\title{
AN ELEMENTARY AND CONSTRUCTIVE SOLUTION TO HILBERT'S 17TH PROBLEM FOR MATRICES
}

\author{
CHRISTOPHER J. HILLAR AND JIAWANG NIE
}

(Communicated by Bernd Ulrich)

\begin{abstract}
We give a short and elementary proof of a theorem of Procesi, Schacher and (independently) Gondard, Ribenboim that generalizes a famous result of Artin. Let $A$ be an $n \times n$ symmetric matrix with entries in the polynomial ring $\mathbb{R}\left[x_{1}, \ldots, x_{m}\right]$. The result is that if $A$ is positive semidefinite for all substitutions $\left(x_{1}, \ldots, x_{m}\right) \in \mathbb{R}^{m}$, then $A$ can be expressed as a sum of squares of symmetric matrices with entries in $\mathbb{R}\left(x_{1}, \ldots, x_{m}\right)$. Moreover, our proof is constructive and gives explicit representations modulo the scalar case.
\end{abstract}

We shall give an elementary proof of the following theorem. Recall that a real matrix is positive semidefinite if it is symmetric with all nonnegative eigenvalues.

Theorem 1. Let $A$ be a symmetric matrix with entries in $\mathbb{R}\left[x_{1}, \ldots, x_{m}\right]$. If $A$ is positive semidefinite for all substitutions $\left(x_{1}, \ldots, x_{m}\right) \in \mathbb{R}^{m}$, then $A$ can be expressed as a sum of squares of symmetric matrices with entries in $\mathbb{R}\left(x_{1}, \ldots, x_{m}\right)$.

This generalizes the following famous result of Artin on nonnegative polynomials; it is the starting point for a large body of work relating positivity and algebra.

Theorem 2 (Artin). If $f \in \mathbb{R}\left[x_{1}, \ldots, x_{m}\right]$ is nonnegative for all substitutions $\left(x_{1}, \ldots, x_{m}\right) \in \mathbb{R}^{m}$, then $f$ is a sum of squares of rational functions in $\mathbb{R}\left(x_{1}, \ldots, x_{m}\right)$.

Theorem 1 was originally proved in [3] and (within a general framework) in [7], although a formulation over a number field was already considered in [2]. Like Artin's result, it guarantees algebraic certificates to (matrix) nonnegativity. However, the known proofs are nonconstructive and employ either model theory [3] or ultraproducts [7]. In contrast, we use only basic facts about real closed fields and linear algebra to give an elegant proof of Theorem 1. Our argument is also explicit, assuming one can construct scalar sums of squares representations in Theorem 2.

Recall that a field $F$ is real if -1 is not a sum of squares in $F$, and a real closed field $R$ is a real field such that any algebraic extension of $R$ that is real must be equal to $R$. Real closed fields have a unique ordering, the nonnegative elements being the squares. For instance, $\mathbb{R}\left(x_{1}, \ldots, x_{m}\right)$ is a real field and $\mathbb{R}$ is real closed. A

\footnotetext{
Received by the editors October 23, 2006 and, in revised form, December 14, 2006.

2000 Mathematics Subject Classification. Primary 12D15, 03C64, 13L05, 14P05, 15A21, $15 \mathrm{~A} 54$.

Key words and phrases. Artin's theorem, Hilbert's 17th problem, sums of squares, positive semidefinite matrix, real closed field.

The first author is supported under an NSF Postdoctoral Research Fellowship. This research was conducted during the Positive Polynomials and Optimization workshop at the Banff International Research Station, October 7-12 (2006), Banff, Canada.
} 
principal minor of a matrix is a determinant of a submatrix determined by the same row and column indices. The set of symmetric matrices over $\mathbb{R}$ with all principal minors nonnegative coincides with the set of positive semidefinite matrices (see for example [4, p. 405]), a fundamental relationship we exploit below. We will prove the following generalization of Theorem 1 to the setting of real fields.

Theorem 3. Let $F$ be a real field and let $A$ be a symmetric matrix with entries in $F$. If the principal minors of $A$ can be expressed as sums of squares in $F$, then $A$ is a sum of squares of symmetric matrices with entries in $F$.

To see how Theorem 1 follows from Theorem 3, consider a principal minor $p\left(x_{1}, \ldots, x_{m}\right) \in \mathbb{R}\left[x_{1}, \ldots, x_{m}\right]$ of the matrix $A$. By assumption, it will be nonnegative for all substitutions $\left(x_{1}, \ldots, x_{m}\right) \in \mathbb{R}^{m}$, and therefore, Artin's theorem implies that it is a sum of squares of rational functions. We may now invoke Theorem 3.

As another application, consider positive semidefinite matrices $A \in \mathbb{Q}^{n \times n}$. Standard matrix theory allows one to write $A=B^{2}$ for a symmetric $B$ with entries that are algebraic numbers; however, Theorem 3 tells us that $A$ is actually a sum of squares of rational matrices. This follows since any nonnegative rational number $a / b=a b / b^{2}$ can be written as a sum of four rational squares by Lagrange's theorem.

To prove Theorem 3, we begin with a lemma. For the basic theory of real closed fields $R$ we will need, we refer the reader to $[5,6]$. The main observation is that a symmetric matrix $A \in R^{n \times n}$ that has all nonnegative principal minors is diagonalizable over $R$ with nonnegative eigenvalues, just as is the case for $\mathbb{R}$.

Lemma 4. Suppose that $A$ is a matrix satisfying the hypotheses of Theorem 3. Then the minimal polynomial $p(t) \in F[t]$ of $A$ is of the form:

$$
p(t)=\sum_{i=0}^{s}(-1)^{s-i} a_{i} t^{i}=t^{s}-a_{s-1} t^{s-1}+\cdots+(-1)^{s} a_{0}
$$

for $a_{i}$ that are sums of squares of elements of $F$. Moreover, $a_{1} \neq 0$.

Proof. Let $p(t)$ be the minimal polynomial of $A$, expressed in the form indicated by (1). We first make the following observation. Let $R$ be any real closure of $F$; this induces an ordering on $R$, in which the principal minors of $A$ are nonnegative (they are sums of squares). Since $A$ is diagonalizable over $R$ and has nonnegative eigenvalues, it follows that each $a_{i} \geq 0$ and also that $p(t)$ has no repeated roots.

Suppose now that some $a_{i}$ was not a sum of squares in $F$. Then there is an ordering of $F$ with $a_{i}$ negative. Let $R$ be a real closure of $F$ that extends the ordering on $F$. By above, $a_{i}$ is nonnegative, a contradiction. To verify the second claim, first notice that $t^{2}$ does not divide $p(t)$ so that $a_{0}$ and $a_{1}$ cannot both be 0 . In a real closure of $F$, the coefficient $a_{1}$ is a sum of products of (nonnegative) roots of $p(t)$. It follows that if $a_{1}=0$, we have $(-1)^{s} a_{0}=p(0)=0$. Thus, $a_{1} \neq 0$.

Proof of Theorem 3. Let $A$ be a symmetric matrix satisfying the hypotheses of Theorem 3. Also, let $p(t)$ be the minimal polynomial for $A$, which has the form prescribed by Lemma 4 . For notational simplicity, we assume that $s$ is odd, although the argument is the same when $s$ is even. Since $p(A)=0$, it follows that

$$
\left(A^{s-1}+a_{s-2} A^{s-3}+\cdots+a_{1} I\right) A=a_{s-1} A^{s-1}+a_{s-3} A^{s-3}+\cdots+a_{0} I .
$$


Set $B=A^{s-1}+\cdots+a_{1} I$, which is invertible (since $a_{1} \neq 0$, in any real closure of $F$, it is diagonalizable with strictly positive eigenvalues). Therefore, we have

$$
A=B \cdot\left(a_{s-1} B^{-2} A^{s-1}+a_{s-3} B^{-2} A^{s-3}+\cdots+a_{0} B^{-2}\right) .
$$

Since $B$ is a sum of squares and $B$ and $B^{-1}$ commute with $A$, the result follows.

Notice that our argument gives a commuting sum of squares representation, the existence of which was also observed in [7]. We close with two examples to illustrate the construction from our proof.

Example 5. The following symmetric matrix is always positive semidefinite:

$$
A=\left[\begin{array}{cc}
1 & x_{1} x_{2} \\
x_{1} x_{2} & 1+x_{1}^{4} x_{2}^{2}+x_{1}^{2} x_{2}^{4}
\end{array}\right] .
$$

However, it is not a sum of squares of matrix polynomials. To see this, let $\mathbf{x}=$ $[1,-1]^{T}$ and suppose that $A$ is a sum of polynomial squares; then so is the polynomial $f\left(x_{1}, x_{2}\right)=\mathbf{x}^{T} A \mathbf{x}=2+x_{1}^{4} x_{2}^{2}+x_{1}^{2} x_{2}^{4}-2 x_{1} x_{2}$. Thus, we can express $f=\sum_{i=1}^{n} p_{i}^{2}$ for some polynomials $p_{i}$ with $\operatorname{deg}\left(p_{i}\right) \leq 3$. Comparing coefficients, $p_{i}$ cannot contain the monomials $x_{1}^{3}, x_{2}^{3}, x_{1}^{2}, x_{2}^{2}, x_{1} x_{2}, x_{1}$, or $x_{2}$ so that we can write $p_{i}=a_{i}+b_{i} x_{1}^{2} x_{2}+c_{i} x_{1} x_{2}^{2}$ for some $a_{i}, b_{i}, c_{i} \in \mathbb{R}$. However, then we cannot produce the term $-2 x_{1} x_{2}$ in $f$, a contradiction. Similarly, $\operatorname{det}(A)$ is not a sum of polynomial squares. It is, however, a sum of rational squares since $\left(x_{1}^{2}+x_{2}^{2}\right) \operatorname{det}(A)$ equals:

$$
\begin{aligned}
\left(x_{2}\right. & \left.-\frac{1}{2} x_{1}^{2} x_{2}\right)^{2}+\left(x_{1}-\frac{1}{2} x_{1} x_{2}^{2}\right)^{2}+2\left(x_{1} x_{2}-\frac{1}{2} x_{1} x_{2}^{3}-\frac{1}{2} x_{1}^{3} x_{2}\right)^{2} \\
& +\frac{3}{4}\left(x_{1}^{2} x_{2}^{4}+x_{1}^{4} x_{2}^{2}\right)+\frac{1}{2}\left(x_{1} x_{2}^{3}+x_{1}^{3} x_{2}\right)^{2} .
\end{aligned}
$$

Since $A^{2}-\operatorname{tr}(A) A+\operatorname{det}(A) I=0$, we have the rational squares representation:

$$
A=\operatorname{tr}(A)\left[\left(\operatorname{tr}(A)^{-1} A\right)^{2}+\operatorname{det}(A)\left(\operatorname{tr}(A)^{-1} I\right)^{2}\right] .
$$

Example 6. The following matrix is positive semidefinite for all substitutions:

$$
A=\left[\begin{array}{ccc}
x_{1}^{2}+2 x_{3}^{2} & -x_{1} x_{2} & -x_{1} x_{3} \\
-x_{1} x_{2} & x_{2}^{2}+2 x_{1}^{2} & -x_{2} x_{3} \\
-x_{1} x_{3} & -x_{2} x_{3} & x_{3}^{2}+2 x_{2}^{2}
\end{array}\right],
$$

but it is not a sum of polynomial squares [1]. Its minimal polynomial has coefficients

$$
\begin{aligned}
& a_{2}=3 x_{3}^{2}+3 x_{2}^{2}+3 x_{1}^{2}, a_{1}=2 x_{2}^{4}+6 x_{1}^{2} x_{3}^{2}+6 x_{1}^{2} x_{2}^{2}+2 x_{1}^{4}+2 x_{3}^{4}+6 x_{2}^{2} x_{3}^{2}, \\
& a_{0}=4 x_{1}^{4} x_{2}^{2}+4 x_{3}^{2} x_{2}^{4}+4 x_{3}^{4} x_{1}^{2}+4 x_{3}^{2} x_{1}^{2} x_{2}^{2},
\end{aligned}
$$

which are all sums of squares. From formula (2), we have

$$
A=\left(A^{2}+a_{1} I\right)\left[a_{2}\left(A+a_{1} A^{-1}\right)^{-2}+a_{0}\left(A^{2}+a_{1} I\right)^{-2}\right] .
$$

We would like to thank Konrad Schmüdgen for discussing this problem with us. 


\section{REFERENCES}

[1] M.-D. Choi, Positive semidefinite biquadratic forms, Lin. Alg. Appl., 12 (1975) 95-100. MR0379365 (52:270)

[2] R. Ciampi, Characterization of a class of matrices as sums of squares, Lin. Alg. Appl., 3 (1970) 45-50. MR0262264 (41:6874)

[3] D. Gondard, P. Ribenboim, Le 17e probleme de Hilbert pour les matrices, Bull. Sci. Math., 98 (1974) 49-56. MR0432613 (55:5600)

[4] R. Horn and C. R. Johnson, Matrix analysis, Cambridge University Press, New York, 1985. MR832183 (87e:15001)

[5] S. Lang, Algebra -3rd ed, Addison-Wesley Publishing Company, New York, 1993. MR0197234 $(33: 5416)$

[6] D. Marker. Model Theory: an Introduction, Springer Verlag, 2002. MR1924282 (2003e:03060)

[7] C. Procesi, M. Schacher, A non-commutative real Nullstellensatz and Hilbert's 17th problem, Ann. of Math., 104 (1976) 395-406. MR0432612 (55:5599)

Department of Mathematics, Texas A\&M University, College Station, Texas 77843

E-mail address: chillar@math.tamu.edu

Institute for Mathematics and its Applications, University of Minnesota, MinneapoLIS, MinnESOTA 55455

E-mail address: njw@ima.umn.edu 